\title{
Evolutionary relationships among salivarius streptococci as inferred from multilocus phylogenies based on I6S rRNA-encoding, recA, secA, and secY gene sequences Jean-François Pombert ${ }^{1}$, Viridiana Sistek ${ }^{2}$, Maurice Boissinot ${ }^{2}$ and Michel Frenette*3
}

Address: ${ }^{1}$ Canadian Institute for Advanced Research, Evolutionary Biology Program, Department of Botany, University of British Columbia, Vancouver, BC, V6T 1Z4, Canada, ${ }^{2}$ Centre de Recherche en Infectiologie de l'Université Laval, Centre Hospitalier Universitaire de Québec (Pavilion CHUL), Quebec City, QC, G1V 4G2, Canada and ${ }^{3}$ Groupe de Recherche en Écologie Buccale, Faculté de Médecine Dentaire, Université Laval, Quebec City, QC, G1V 0A6, Canada

Email: Jean-François Pombert - jpombert@interchange.ubc.ca; Viridiana Sistek - Viridiana.Sistek@crchul.ulaval.ca; Maurice Boissinot - Maurice.Boissinot@crchul.ulaval.ca; Michel Frenette* - Michel.Frenette@greb.ulaval.ca

* Corresponding author

Published: 30 October 2009

BMC Microbiology 2009, 9:232 doi:10.1 186/147/-2180-9-232
Received: 9 April 2009

Accepted: 30 October 2009

This article is available from: http://www.biomedcentral.com//47I-2/80/9/232

(c) 2009 Pombert et al; licensee BioMed Central Ltd.

This is an Open Access article distributed under the terms of the Creative Commons Attribution License (http://creativecommons.org/licenses/by/2.0), which permits unrestricted use, distribution, and reproduction in any medium, provided the original work is properly cited.

\begin{abstract}
Background: Streptococci are divided into six phylogenetic groups, i.e, anginosus, bovis, mitis, mutans, pyogenic, and salivarius, with the salivarius group consisting of only three distinct species. Two of these species, Streptococcus salivarius and Streptococcus vestibularis, are members of the normal human oral microflora whereas the third, Streptococcus thermophilus, is found in bovine milk. Given that S. salivarius and S. vestibularis share several physiological characteristics, in addition to inhabiting the same ecosystem, one would assume that they would be more closely related to each other than to $S$. thermophilus. However, the few phylogenetic trees published so far suggest that $S$. vestibularis is more closely related to $S$. thermophilus. To determine whether this phylogenetic relationship is genuine, we performed phylogenetic inferences derived from secA and secY, the general secretion housekeeping genes, recA, a gene from a separate genetic locus that encodes a major component of the homologous recombinational apparatus, and I6S rRNA-encoding gene sequences using other streptococcal species as outgroups.
\end{abstract}

Results: The maximum likelihood (ML) and maximum parsimony (MP) phylogenetic inferences derived from the secA and recA gene sequences provided strong support for the $S$. vestibularis/S. thermophilus sister-relationship, whereas $16 \mathrm{~S}$ rRNA-encoding and secY-based analyses could not discriminate between alternate topologies. Phylogenetic analyses derived from the concatenation of these sequences unambiguously supported the close affiliation of $S$. vestibularis and $S$. thermophilus.

Conclusion: Our results corroborated the sister-relationship between $S$. vestibularis and $S$. thermophilus and the concomitant early divergence of $S$. salivarius at the base of the salivarius lineage.

\section{Background}

The Streptococcus genus comprises ninety-two recognized species that are present in a wide variety of habitats [1]. In humans and animals, a number of streptococcal species are important pathogens (e.g., S. pneumoniae, S. pyogenes, $S$. suis, and $S$. mutans), while others are members of mutu- 
alistic microflora (e.g., S. oralis, S. downei, S. dentirousetti, and S. salivarius). The species of the Streptococcus genus have been divided into six groups (anginosus, bovis, mitis, mutans, pyogenic, and salivarius) based on $16 \mathrm{~S}$ rDNA phylogenetic inferences [2]. According to these authors, the salivarius group is composed of three species: (1) S. salivarius, a pioneer colonizer of the human oral mucosa that is isolated mainly from the dorsum of the tongue, the cheeks, and the palate [3], (2) S. vestibularis, a mutualistic bacterium that is present on the vestibulum of the human oral mucosa [4], and (3) S. thermophilus, a thermophilic species [5] that is part of starter cultures used in the production of yogurt and Swiss- or Italian-type cooked cheeses. Unlike S. salivarius and S. vestibularis, $S$. thermophilus is not a natural inhabitant of the human oral mucosa and is commonly found on the mammary mucosa of bovines, its natural ecosystem, as inferred from its presence and that of thermophilus-specific bacteriophages in raw milk isolates [6-8].

The common ecosystem is not the only feature shared by $S$. salivarius and $S$. vestibularis. Biochemical investigations of functional metabolic pathways have revealed that these two species share a high level of physiological resemblance. For example, S. salivarius and S. vestibularis are capable of hydrolyzing esculin and generating acidic compounds from maltose and $N$-acetyl-glucosamine, while $S$. thermophilus is not ([9] and references therein). Both S. salivarius and $S$. vestibularis are also opportunistic pathogens that can cause mild to severe infective endocarditis [10$12]$, whereas $S$. thermophilus has never been implicated in such infections. Given the home environments of the organisms, the high level of metabolic similarity between $S$. salivarius and $S$. vestibularis, and the more restricted spectrum of carbon sources that can be used by $S$. thermophilus [13], one would assume that $S$. salivarius and $S$. vestibularis would be more related to each other than to $S$. thermophilus. However the few phylogenetic trees published so far that include all three species, as inferred from $16 S$ rRNA-encoding gene sequences [2] and the housekeeping gene sodA that encodes the manganese-dependent superoxide dismutase [14], suggest that a schism generated $S$. vestibularis and $S$. thermophilus subsequent to the early divergence of $S$. salivarius. However, since these two phylogenetic studies $[2,14]$ were limited to only one taxon for each species, the inferred relationships between these three species might be inaccurate.

To investigate the evolutionary relationships between the three species making up the salivarius group, we performed phylogenetic inferences based on the 16S rRNAencoding, secA and $\sec Y$ housekeeping genes and the important yet non-essential recA gene using an identical distribution of streptococcal strains among the various markers to facilitate direct comparisons and allow the concatenation of the individual sequences into a single matrix. These four ubiquitous genes are widely distributed and have homologues in all three kingdoms, i.e., Bacteria, Archaea, and Eukarya (for reviews see [15-17]). The 16S rRNA-encoding gene, which codes for the major ribonucleic constituent of the bacterial small ribosomal subunit [18], is one the most frequently used housekeeping phylogenetic markers [19], while the secA and secY genes code for components of the general protein-secretion pathway, which is essential for several cell functions [20]. The fourth gene, $r e c A$, codes for a product that initiates the formation of Holliday junction intermediates during homologous recombination [21]. Our ML and MP phylogenetic inferences based of these four gene sequences are in agreement with earlier findings by Kawamura et al. [2] and Poyart et al. [14] and corroborate the S. thermophilus/S. vestibularis sister-relationship.

\section{Results \\ Phylogenetic analyses of secA gene sequences}

We began our investigation of the branching order of the streptococci of the salivarius group by looking at phylogenetic trees inferred from the secA gene (Figure 1). As expected, the salivarius group comprising $S$. salivarius, $S$. thermophilus, and $S$. vestibularis was monophyletic in all the ML and MP bootstrap replicates. The S. thermophilus and $S$. vestibularis species monophylies were strongly supported by the ML and MP analyses, while support for the $S$. salivarius monophyly ranged from weak to moderate in the ML analyses and strong in the MP analyses. Our phylogenetic analyses based on secA gene sequences strongly support the notion that $S$. vestibularis and $S$. thermophilus are closely related species. The node comprising these two species was retrieved in all the ML and MP bootstrap replicates, while the other two possible alternate topologies, i.e., the S. salivarius/S. vestibularis and S. salivarius/S. thermophilus relationships, were not recovered in any of the replicates.

\section{Phylogenetic analyses of secY gene sequences}

The ML and MP phylogenetic inferences derived from the secY gene were not as conclusive (Figure 2). Although the monophyly of the salivarius group was again recovered in all the bootstrap replicates, together with the unambiguous delineation of the $S$. vestibularis and $S$. thermophilus species, the $S$. salivarius species was paraphyletic, with $S$. salivarius strain CCRI 17393 branching out at the base of the three $S$. thermophilus strains. However, given the differences in branch lengths between $S$. salivarius strain CCRI 17393 and the other $S$. salivarius strains, the positioning of this strain at the base of the $S$. thermophilus strains appears dubious and may result from artifactual attraction between locally long branches, an effect that might have been exacerbated by the scarcity of informative characters in this dataset. Of the 1287 positions constituting the $\sec Y$ dataset, 135 displayed variations between members of the salivarius group, with only 98 being phylogenetically 


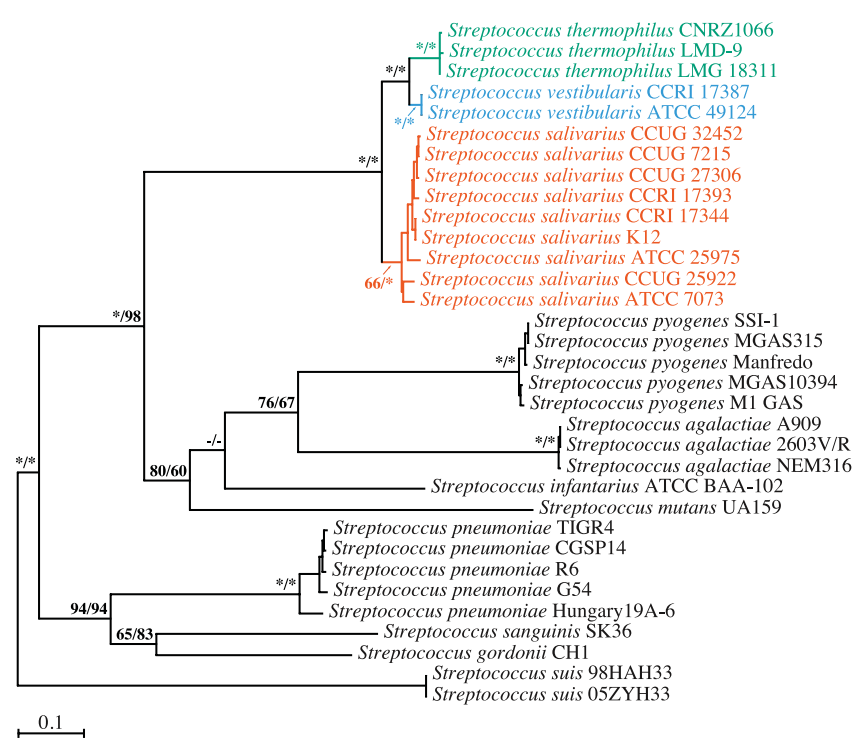

Figure I

Branching order of members of the salivarius group as inferred from ML and MP analyses of secA gene sequences ( 2484 positions; I 26 I variable, I I 69 phylogenetically informative). The best $M L$ tree computed with PHYML 3.0 under the GTR $+\Gamma 4+I$ model of nucleotide substitution is shown here. Bootstrap support for the major nodes is indicated over the corresponding nodes: ML values left, MP values right. Asterisks denote nodes that were retrieved in all the bootstrap replicates. Dashes indicate nodes that were retrieved in fewer than $50 \%$ of the bootstrap replicates. Streptococcal species belonging to the salivarius group are shown in orange (S. salivarius), blue ( $S$. vestibularis), or green (S. thermophilus). Strains CCUG 7215 and CCUG 27306, which are categorized as Streptococcus vestibularis in the CCUG culture collection, are capable of using raffinose as the sole carbon source. This contradicts Whiley and Hardie's [4] canonical S. vestibularis species definition. This metabolic trait is more a hallmark of the closely related Streptococcus salivarius species, to which the two strains belong. Other streptococcal species shown in black were outgroups. Branch lengths are drawn to scale.

informative (Table 1). In contrast, the secA dataset featured 266 variable sites, with 222 phylogenetically informative characters among members of the salivarius group, i.e., more than twice the amount of potentially discriminating information. On the other hand, we cannot exclude the possibility that the branching of $S$. salivarius strain CCRI 17393 at the base of the S. thermophilus strains in our secY-based analyses resulted from a genuine phylogenetic signal. If this is true, then the secA and secY gene sequences from $S$. salivarius strain CCRI 17393 have evolved in different directions. In any event, the phylogenetic resolution of the $\sec Y$ dataset was not sufficient to unambiguously infer the branching order between the three species making up the salivarius group.

\section{Phylogenetic analyses of recA partial gene sequences}

Our phylogenetic inferences based on recA partial gene sequences yielded clearer insights into the branching order of the members of the salivarius group (Figure 3 ), which were clustered together in all the ML and MP bootstrap replicates, while the two $S$. vestibularis strains formed a united clade in all the replicates, and the three $S$. thermophilus strains branched together in the vast majority of the bootstrap replicates. The monophyly of the S. salivarius species was recovered in $98 \%$ of the MP bootstrap replicates, although ML-based phylogenetic inferences could not discriminate between paraphyletic and monophyletic S. salivarius clades (52\% vs. $48 \%$ of the bootstrap replicates, respectively). Like the secA-based phylogenetic inferences, the analyses derived from the reca gene sequences strongly supported a sister-relationship between the S. vestibularis and S. thermophilus species. The node comprising these two species was robust and was recovered in all the ML and MP bootstrap replicates.

\section{Phylogenetic analyses of I 6S rRNA-encoding gene sequences}

Building on the phylogeny published by Kawamura et al. [2], we reinvestigated the branching order among the salivarius streptococci using 16S rRNA-encoding gene sequences and expanded taxon sampling within the salivarius group. As can be seen in Figure 4, even though the salivarius group was recovered in all the bootstrap replicates, the branching order within this taxonomic entity was not well defined. Of the three species, only $S$. thermophilus composed a monophyletic assemblage. The other two, $S$. vestibularis and $S$. salivarius, were not resolved. This contrasted with the results obtained by Kawamura et al. [2], who reported that the S. vestibularis and $S$. thermophilus species branched together with strong bootstrap support. It should be noted, however, that the 16S rRNA-encoding gene sequences exhibited almost no variability among salivarius streptococci. Of the 1374 positions making up our 16S rRNA-encoding gene dataset, only 14 were variable, with a mere eight positions giving tangible phylogenetic information for the three salivarius species (Table 1). The topologies inferred from the 16S rRNA-encoding gene sequences should thus be treated with caution with respect to the branching order of salivarius streptococci.

\section{Phylogenetic analyses of concatenated gene sequences}

To increase the resolving power of our phylogenetic analyses, we concatenated the four previous datasets into a single matrix to pool their phylogenetic signals. As anticipated, our ML and MP analyses based on the concatenated $\sec A, \sec Y$, recA, and 16S rRNA-encoding gene sequences yielded superior resolved topologies (Figure 5). While the clade constituting the salivarius group and the monophylies of the $S$. thermophilus and $S$. vestibularis species were once again recovered in all of the bootstrap replicates, support for the monophyly of the $S$. salivarius spe- 
Table I: Main features of each phylogenetic dataset

\begin{tabular}{lccccc}
\hline & & \multicolumn{2}{c}{ Full Dataset } & \multicolumn{2}{c}{ Salivarius Subset $^{c}$} \\
\cline { 3 - 6 } Name & Length & Variable $^{\mathbf{a}}$ & Informative $^{\mathbf{b}}$ & Variable $^{\mathbf{a}}$ & Informative $^{\mathbf{b}}$ \\
\hline $\sec A$ & 2484 & 1261 & 1169 & 266 & 222 \\
$\sec Y$ & 1287 & 735 & 686 & 135 & 98 \\
$\operatorname{rec} A$ & 798 & 309 & 289 & 102 & 96 \\
I6S & 1374 & 169 & 141 & 517 & 8 \\
Alld & 5943 & 2474 & 2285 & 424 \\
\hline
\end{tabular}

a Number of variable characters

b Number of phylogenetically informative characters

c Values observed between the $14 \mathrm{~S}$. salivarius, S. thermophilus, and S. vestibularis taxa

Dataset containing the I6S rRNA-encoding, $\operatorname{rec} A, \sec A$, and $\sec Y$ concatenated gene sequences

cies increased appreciably. In the ML analyses, the concatenation of the various datasets had a synergistic effect on the S. salivarius monophyly for which bootstrap support attained a level not seen with any of the independent gene datasets. In the MP analyses, the bootstrap support for this monophyly remained strong. The phylogenetic inferences derived from the concatenated $\sec A$, $\sec Y$, recA, and 16S rRNA-encoding gene sequences

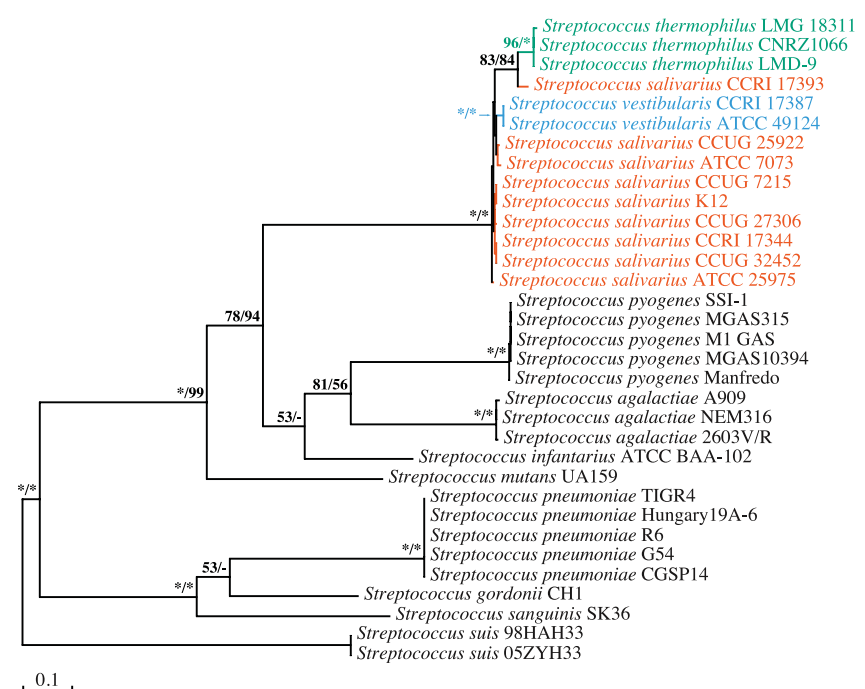

Figure 2

Branching order of members of the salivarius group as inferred from $M L$ and MP analyses of sec $Y$ gene sequences ( 1287 positions; 735 variable, 686 phylogenetically informative). The best ML tree computed with PHYML 3.0 under the GTR $+\Gamma 4+I$ model of nucleotide substitution is shown here. Bootstrap support for the major nodes is indicated over the corresponding nodes: ML values left, MP values right. Asterisks denote nodes that were retrieved in all the bootstrap replicates. Dashes indicate nodes that were retrieved in fewer than $50 \%$ of the bootstrap replicates. Streptococcal species belonging to the salivarius group are shown in orange (S. salivarius), blue (S. vestibularis) or green (S. thermophilus). Other streptococcal species shown in black were outgroups. Branch lengths are drawn to scale. strongly supported the sister-relationship between the $S$. vestibularis and $S$. thermophilus species. This sister-relationship and the concomitant early divergence of the S. salivarius species at the base of the salivarius clade were recovered in $100 \%$ and $98 \%$ of the ML and MP bootstrap replicates, respectively.

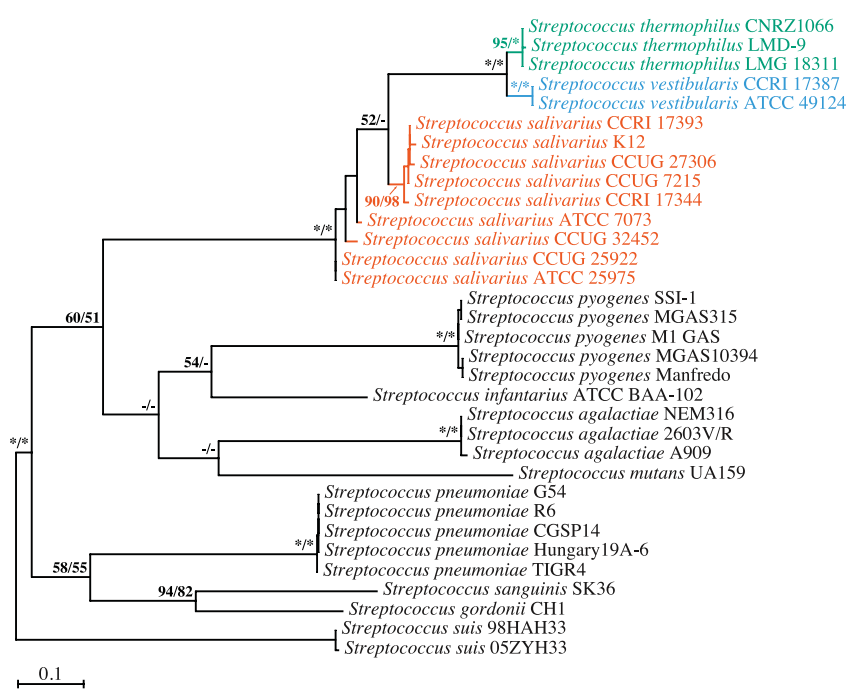

Figure 3

Branching order of members of the salivarius group as inferred from ML and MP analyses of recA partial gene sequences (798 positions; 309 variable, 289 phylogenetically informative). The best $M L$ tree computed with PHYML 3.0 under the GTR $+\Gamma 4+I$ model of nucleotide substitution is shown here. Bootstrap support for the major nodes is indicated over the corresponding nodes: ML values left, MP values right. Asterisks denote nodes that were retrieved in all the bootstrap replicates. Dashes indicate nodes that were retrieved in fewer than $50 \%$ of the bootstrap replicates. Streptococcal species belonging to the salivarius group are shown in orange (S. salivarius), blue ( $S$. vestibularis) or green (S. thermophilus). Other streptococcal species shown in black were outgroups. Branch lengths are drawn to scale. 


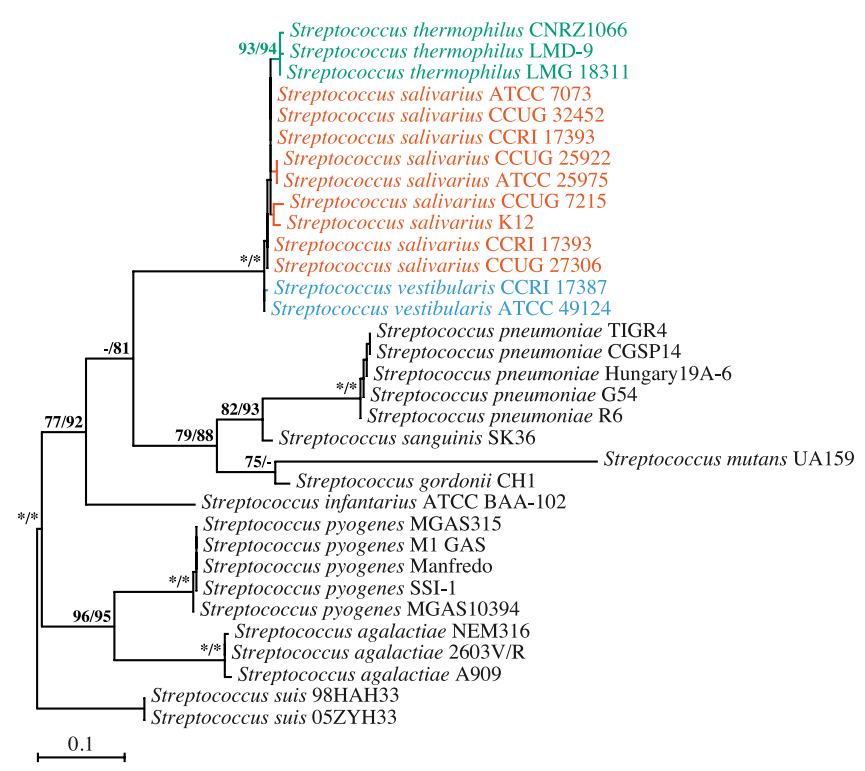

Figure 4

Branching order of members of the salivarius group as inferred from ML and MP analyses of I6S rRNAencoding partial gene sequences (I 374 positions; 169 variable, I4I phylogenetically informative). The best ML tree computed with PHYML 3.0 under the GTR $+\Gamma 4+1$ model of nucleotide substitution is shown here. Bootstrap support for the major nodes is indicated over the corresponding nodes: ML values left, MP values right. Asterisks denote nodes that were retrieved in all the bootstrap replicates. Dashes indicate nodes that were retrieved in fewer than $50 \%$ of the bootstrap replicates. Streptococcal species belonging to the salivarius group are shown in orange (S. salivarius), blue (S. vestibularis), or green (S. thermophilus). Other streptococcal species shown in black were outgroups.

Branch lengths are drawn to scale.

\section{Discussion}

When we began our study, we expected that the S. salivarius and $S$. vestibularis species would be more closely related to each other given their level of physiological resemblance and that the $S$. vestibularis/S. thermophilus sisterrelationship inferred in previous phylogenetic studies $[2,14]$ would not be robustly supported. Obviously, this was not the case. Our results were in complete agreement with earlier neighbor-joining phylogenies based on partial 16S rRNA-encoding and sodA gene sequences $[2,14]$ and corroborated the $S$. vestibularis/S. thermophilus sisterrelationship. This sister-relationship was not dependent on the method of phylogenetic reconstruction and was strongly supported by both our ML and MP analyses. Furthermore, while the 16S-rRNA-encoding and secY gene sequences were unable to discriminate between the $S$. vestibularis/S. thermophilus and the alternate $S$. vestibularis/S. salivarius and $S$. salivarius/S. thermophilus sister-relationships, we observed no serious incongruities between the

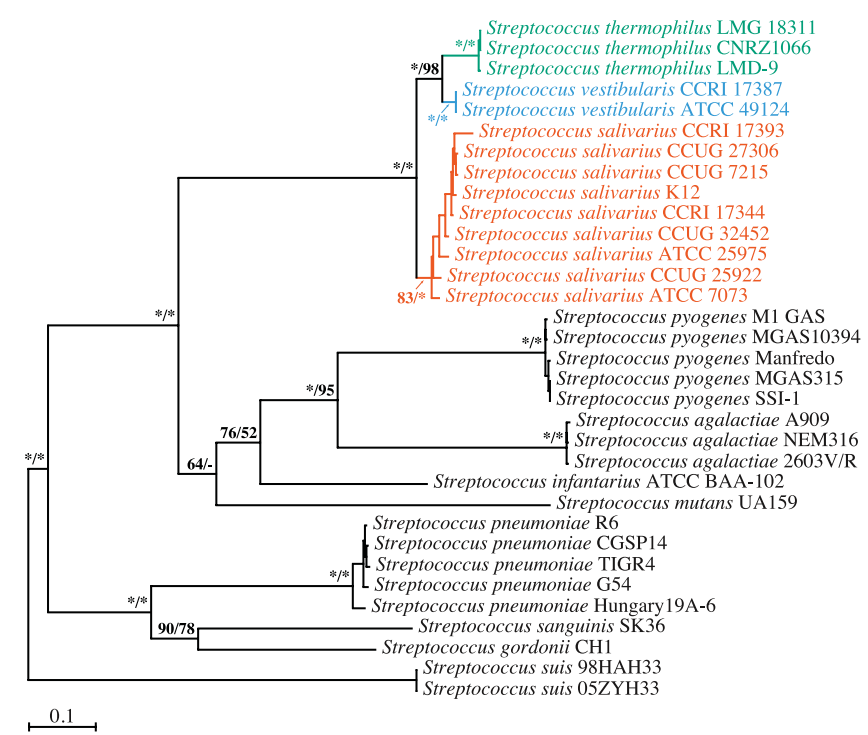

Figure 5

Branching order of members of the salivarius group as inferred from ML and MP analyses of concatenated I6S rRNA-encoding, recA, secA, and secY gene sequences (5943 positions; 2474 variable, 2285 phylogenetically informative). The best $M L$ tree computed with PHYML 3.0 under the GTR $+\Gamma 4+I$ model of nucleotide substitution is shown here. Bootstrap support for the major nodes is indicated over the corresponding nodes: ML values left, MP values right. Asterisks denote nodes that were retrieved in all the bootstrap replicates. Dashes indicate nodes that were retrieved in fewer than $50 \%$ of the bootstrap replicates. Streptococcal species belonging to the salivarius group are shown in orange (S. salivarius), blue ( $S$. vestibularis) or green (S. thermophilus). Other streptococcal species shown in black were outgroups. Branch lengths are drawn to scale.

topologies inferred from these molecular markers and those inferred from the recA and secA gene sequences.

The S. vestibularis/S. thermophilus sister-relationship inferred from our phylogenetic analyses is not necessarily incompatible with the observation that $S$. vestibularis share more phenotypic similarities with $S$. salivarius than with $S$. thermophilus. Following speciation from a putative common ancestor physiologically similar to S. salivarius, the two newly formed species could have evolved differently, with $S$. vestibularis and $S$. thermophilus independently retaining and discarding a number of ancestral features. Many of the phenotypic losses observed in the $S$. thermophilus species could have been induced by its adaptation to its new ecosystem, i.e., the bovine mammary mucosa. In particular, because this species has access to a wealth of nutrients within bovine milk, polyvalence for sugar metabolism-related genes might not be as important for this species as for its relatives inhabiting the 
Table 2: Accession numbers

\begin{tabular}{|c|c|c|c|c|}
\hline \multirow[b]{2}{*}{ Streptococci } & \multicolumn{4}{|c|}{ Accession Numbers } \\
\hline & recA & $\sec A$ & $\sec Y$ & I6S rDNA \\
\hline $\begin{array}{l}\text { S. agalactiae } \\
2603 \mathrm{~V} / \mathrm{R}\end{array}$ & [GenBank: NC 004116] & [GenBank: NC 004116$]$ & [GenBank: NC 004II6] & [GenBank: NC 004116] \\
\hline $\begin{array}{l}\text { S. agalactiae } \\
\text { A909 }\end{array}$ & [GenBank: NC 007432] & [GenBank: NC 007432] & [GenBank: NC 007432] & [GenBank: NC 007432] \\
\hline $\begin{array}{l}\text { S. agalactiae } \\
\text { NEM } 316\end{array}$ & [GenBank: NC 004368] & [GenBank: NC 004368] & [GenBank: NC 004368] & [GenBank: NC 004368] \\
\hline $\begin{array}{l}\text { S. gordonii str. } \\
\text { Challis substr. } \mathrm{CHI}\end{array}$ & [GenBank: NC 009785] & [GenBank: NC 009785] & [GenBank: NC 009785] & [GenBank: NC 009785] \\
\hline $\begin{array}{l}\text { S. infantarius } \\
\text { ATCC BAA-102 }\end{array}$ & $\begin{array}{l}\text { [GenBank: } \\
\text { NZ ABJK020000I5] }\end{array}$ & $\begin{array}{l}\text { [GenBank: } \\
\text { NZ ABJK02000019] }\end{array}$ & $\begin{array}{l}\text { [GenBank: } \\
\text { NZ AB|K020000।3] }\end{array}$ & [GenBank: AF429762] \\
\hline $\begin{array}{l}\text { S. mutans } \\
\text { UAI59 }\end{array}$ & [GenBank: NC 004350] & [GenBank: NC 004350] & [GenBank: NC 004350] & [GenBank: NC 004350] \\
\hline $\begin{array}{l}\text { S. pneumoniae } \\
\text { CGSPI4 }\end{array}$ & [GenBank: NC 010582] & [GenBank: NC 010582$]$ & [GenBank: NC 010582$]$ & [GenBank: NC 010582] \\
\hline $\begin{array}{l}\text { S. pneumoniae } \\
\text { G54 }\end{array}$ & [GenBank: NC 011 072] & [GenBank: NC 011072$]$ & [GenBank: NC 011072$]$ & [GenBank: NC 011 072] \\
\hline $\begin{array}{l}\text { S. pneumoniae } \\
\text { Hungary19A-6 }\end{array}$ & [GenBank: NC 010380$]$ & [GenBank: NC 010380$]$ & [GenBank: NC 010380$]$ & [GenBank: NC 010380$]$ \\
\hline $\begin{array}{l}\text { S. pneumoniae } \\
\text { R6 }\end{array}$ & [GenBank: NC 003098] & [GenBank: NC 003098] & [GenBank: NC 003098] & [GenBank: NC 003098] \\
\hline $\begin{array}{l}\text { S. pneumoniae } \\
\text { TIGR4 }\end{array}$ & [GenBank: NC 003028] & [GenBank: NC 003028] & [GenBank: NC 003028] & [GenBank: NC 003028] \\
\hline $\begin{array}{l}\text { S. pyogenes MI } \\
\text { GAS }\end{array}$ & [GenBank: NC 002737] & [GenBank: NC 002737] & [GenBank: NC 002737] & [GenBank: NC 002737] \\
\hline $\begin{array}{l}\text { S. pyogenes } \\
\text { MGASI0394 }\end{array}$ & [GenBank: NC 006086] & [GenBank: NC 006086] & [GenBank: NC 006086] & [GenBank: NC 006086] \\
\hline $\begin{array}{l}\text { S. pyogenes } \\
\text { MGAS3I5 }\end{array}$ & [GenBank: NC 004070] & [GenBank: NC 004070] & [GenBank: NC 004070$]$ & [GenBank: NC 004070] \\
\hline $\begin{array}{l}\text { S. pyogenes } \\
\text { SSI-I }\end{array}$ & [GenBank: NC 004606] & [GenBank: NC 004606] & [GenBank: NC 004606] & [GenBank: NC 004606] \\
\hline $\begin{array}{l}\text { S. pyogenes str. } \\
\text { Manfredo }\end{array}$ & [GenBank: NC 009332] & [GenBank: NC 009332] & [GenBank: NC 009332] & [GenBank: NC 009332] \\
\hline $\begin{array}{l}\text { S. salivarius } \\
\text { ATCC } 25975\end{array}$ & [GenBank: F]154806'] & [GenBank: F]1548I7b] & [GenBank: F]154828b] & [GenBank: F]154797b] \\
\hline $\begin{array}{l}\text { S. salivarius } \\
\text { ATCC } 7073\end{array}$ & [GenBank: FJI54807b] & [GenBank: F]154818b] & [GenBank: FJI54829b] & [GenBank: AYI88352] \\
\hline $\begin{array}{l}\text { S. salivarius } \\
\text { CCRI I } 7344\end{array}$ & [GenBank: F]I54808º ] & [GenBank: F]I54819b] & [GenBank: F]I54830 b ] & [GenBank: F]I54798b] \\
\hline $\begin{array}{l}\text { S. salivarius } \\
\text { CCRI I } 7393\end{array}$ & [GenBank: F]/54809b] & [GenBank: F]I54820ㄷ] & [GenBank: F]I5483|b] & [GenBank: F]I54799b] \\
\hline $\begin{array}{l}\text { S. salivarius } \\
\text { CCUG } 25922\end{array}$ & [GenBank: F]154810º] & [GenBank: F]I5482/b] & [GenBank: F]I54832'] & [GenBank: F]I54800 ${ }^{\mathrm{b}}$ ] \\
\hline $\begin{array}{l}\text { S. salivarius } \\
\text { CCUG 27306a }\end{array}$ & [GenBank: F]I548IIb] & [GenBank: F]I54822 ${ }^{\mathrm{b}}$ ] & [GenBank: FJI54833'] & [GenBank: FJI5480I'b] \\
\hline $\begin{array}{l}\text { S. salivarius } \\
\text { CCUG } 32452\end{array}$ & [GenBank: F]I548I2b] & [GenBank: F]I54823ㄷ] & [GenBank: F]I54834b] & [GenBank: F]I54802 ${ }^{\mathrm{b}}$ ] \\
\hline $\begin{array}{l}\text { S. salivarius } \\
\text { CCUG } 7215^{a}\end{array}$ & 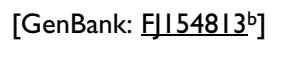 & [GenBank: F]I54824ㄴ] & [GenBank: F]154835'] & [GenBank: F]I54803'] \\
\hline $\begin{array}{l}\text { S. salivarius } \\
\mathrm{K} 12\end{array}$ & [GenBank: FJI548|4b] & [GenBank: FJI54825ㄷ] & [GenBank: FJI54836º] & [GenBank: F]I54804b] \\
\hline $\begin{array}{l}\text { S. sanguinis } \\
\text { SK36 }\end{array}$ & [GenBank: NC 009009] & [GenBank: NC 009009] & [GenBank: NC 009009] & [GenBank: NC 009009] \\
\hline $\begin{array}{l}\text { S. suis } \\
\text { 05ZYH33 }\end{array}$ & [GenBank: NC 009442] & [GenBank: NC 009442] & [GenBank: NC 009442] & [GenBank: NC 009442] \\
\hline $\begin{array}{l}\text { S. suis } \\
\text { 98HAH33 }\end{array}$ & [GenBank: NC 009443] & [GenBank: NC 009443] & [GenBank: NC 009443] & [GenBank: NC 009443] \\
\hline $\begin{array}{l}\text { S. thermophilus } \\
\text { CNRZ1066 }\end{array}$ & [GenBank: NC 006449] & [GenBank: NC 006449] & [GenBank: NC 006449] & [GenBank: NC 006449] \\
\hline $\begin{array}{l}\text { S. thermophilus } \\
\text { LMD-9 }\end{array}$ & [GenBank: NC 008532] & [GenBank: NC 008532] & [GenBank: NC 008532] & [GenBank: NC 008532] \\
\hline
\end{tabular}


Table 2: Accession numbers (Continued)

\begin{tabular}{|c|c|c|c|c|}
\hline $\begin{array}{l}\text { S. thermophilus } \\
\text { LMG I831। }\end{array}$ & [GenBank: NC 006448] & [GenBank: NC 006448] & [GenBank: NC 006448] & [GenBank: NC 006448 ] \\
\hline $\begin{array}{l}\text { S. vestibularis } \\
\text { ATCC } 49124\end{array}$ & [GenBank: F]I54815'] & [GenBank: F]I54826 ${ }^{\mathrm{b}}$ ] & [GenBank: F]I54837b] & [GenBank: AY|88353] \\
\hline $\begin{array}{l}\text { S. vestibularis } \\
\text { CCRI I } 7387\end{array}$ & [GenBank: F]154816 ${ }^{\mathrm{b}}$ ] & [GenBank: F]I54827b] & [GenBank: F]I54838 b ] & [GenBank: F]I54805'] \\
\hline
\end{tabular}

a Erroneously categorized as Streptococcus vestibularis in the culture collection of the University of Göteborg (CCUG)

b This study

human oral mucosa [13]. Further losses could have been caused by additional selective pressure applied on $S$. thermophilus commercial strains ([22] and references therein) that are used in the manufacture of various dairy products.

The relationships inferred among the three salivarius streptococci raise interesting questions regarding their establishment in their respective ecosystems. Because the $S$. salivarius/S. vestibularis sister-relationship is not supported by phylogenetic analyses, the colonization of the human oral cavity by an ancestor of $S$. thermophilus present in bovine milk, which would have then speciated over time into S. salivarius and S. vestibularis, is not plausible. Furthermore, the independent colonization of bovine mammary and human oral mucosae by a putative ancestor originating from a third environment is not compatible with these phylogenies unless we assume two distinct yet closely related streptococcal ancestors; one that independently colonized the two ecosystems yielding $S$. thermophilus and $S$. vestibularis on the one hand, and $S$. salivarius on the other. Alternatively, the direct or indirect invasion of the bovine mammary mucosa by an ancestor of $S$. vestibularis originating from the human oral cavity would also be compatible with the S. vestibularis/S. thermophilus sister-relationship.

\section{Conclusion}

The phylogenetic analyses presented in the present paper strongly support the $S$. vestibularis/S. thermophilus sisterrelationship and the concomitant early divergence of $S$. salivarius at the base of the salivarius clade, which is in agreement with previous $16 \mathrm{~S} \mathrm{rDNA} /$ sodA-based phylogenetic inferences $[2,14]$. One of the main reasons for conducting the present study was the paucity of phylogenetic studies involving all three species making up the salivarius group. Although a number of studies that included $S$. salivarius and $S$. vestibularis have been published, $S$. thermophilus has been omitted more often than not since it is not retrieved from human clinical isolates. Since the complete genome sequences of three $S$. thermophilus strains are now available, it would be interesting to revisit phylogenetic studies that involve different phylogenetic markers and $S$. salivarius/S. vestibularis but not $S$. thermophilus to verify whether the addition of $S$. thermophilus would result in a similar branching order among salivarius streptococci.

\section{Methods}

\section{Source organisms}

Streptococcus salivarius strains ATCC 7073 and 25975 and Streptococcus vestibularis strain ATCC 49124 were obtained from the American Type Culture Collection (Manassas, VA, USA). Streptococcus salivarius strain K12 was obtained from BLIS Technologies Ltd. (Dunedin, New Zealand). Streptococcus salivarius strains CCUG 32452 and 25922 and Streptococcus vestibularis strains CCUG 7215 and 27306 (renamed S. salivarius strains CCUG 7215 and 27306 herein) were obtained from the University of Göteborg Culture Collection (Göteborg, Sweden). Streptococcus salivarius clinical isolates CCRI 17344 and CCRI 17393 and Streptococcus vestibularis clinical isolate CCRI 17387 were obtained from the Centre de Recherche en Infectiologie of the Centre Hospitalier Universitaire de Québec (CHUQ), CHUL Pavilion (Quebec City, QC, Canada). The identity of the $S$. vestibularis strains was confirmed by comparative growth on TYE medium containing either raffinose or glucose as the sole carbon source.

\section{DNA isolation and sequencing}

Streptococcal strains were grown in TYE-glucose liquid medium as described in Lévesque et al. [23] or on sheepblood agar medium overnight at $35^{\circ} \mathrm{C}$ in a $5 \% \mathrm{CO}_{2}$ atmosphere. Their 16S rRNA-encoding, recA, secA, and $\sec Y$ genes were amplified by polymerase chain reaction (PCR) from either (A) purified chromosomal DNA, (B) DNA released from boiled bacterial colonies, or (C) bacterial lysates. Purified chromosomal DNA was obtained as follows. Streptococcal cells were pelleted by centrifugation. The pellets were washed for $30 \mathrm{~min}$ at $37^{\circ} \mathrm{C}$ in 50 $\mathrm{mM}$ Tris-HCl buffer ( $\mathrm{pH}$ 8) containing 6.7\% (w/v) sucrose, $1 \mathrm{mM}$ EDTA, and $40 \mathrm{U} / \mathrm{ml}$ of mutanolysin. SDS (final concentration 1\%) was then added and the cells were lysed for $10 \mathrm{~min}$ at $60^{\circ} \mathrm{C}$. Proteinase $\mathrm{K}$ (final concentration $0.14 \mathrm{mg} / \mathrm{ml}$ ) was added and the incubation was continued for an additional $20 \mathrm{~min}$. Chromosomal DNA was isolated from the cellular debris using the standard phenol/ChCl ${ }_{3}$ extraction protocol described by Sambrook et al. [24]. DNA released from boiled cells was obtained as follows. Streptococcal colonies grown on TYE-glucose agar or blood agar medium were suspended in $100 \mu \mathrm{l}$ of distilled water and then boiled at $94^{\circ} \mathrm{C}$ for $3 \mathrm{~min}$. This suspension was then used instead of sterile distilled water in the PCR protocols. Bacterial lysates were obtained with 
the BD GeneOhm ${ }^{\mathrm{TM}}$ Lysis Kit (BD Diagnostics-GeneOhm, Quebec City, QC, Canada). The 16S rRNA-encoding, recA, $\sec A$ and $\sec Y$ genes were amplified by PCR using primers 16S_F (5'-AGTTTGATCCTGGCTCAGGACG-3') and 16S_R (5'-ATCCAGCCGCACCTTCCGATAC-3'), SSU27 (5'-AGAGTTTGATCMTGGCTCAG-3') and SSU1492 (5'TACGGYTACCTTGTTACGACTT-3'), RStrGseq81 (5'GAAAWWIATYGARAAAGAITTTGGTAA-3') and RStrGseq937 (5'-TTYTCAGAWCCTTGICCAATYTTYTC3'), SecAAMON (5'-CAGGCCTTTGAAAATCTCTTAC-3') and SecAAVAL (5'-CTCTTTATCACGAGCTTGCTTC-3'), or SecYAMON (5'-CTGCTGAAGCAGCTATCACTGC-3') and SecYAVAL (5'-CTTTACCAGCACCTGGTAGACC-3'). The PCR templates were sequenced using Sanger dideoxynucleotide chemistry as described in Pombert et al. [25]. The sequences were edited and assembled using STADEN package version 1.7.0 http://staden.sourceforge.net/ or SEQUENCHER 4.8 (GeneCodes, Ann Arbor, MI, USA).

\section{Dataset preparation}

The sequences we used were either retrieved from GenBank or sequenced by the authors. Sequences showing ambiguous base calling in databases were not selected for phylogenetic analyses. The 16S rRNA-encoding gene sequences were aligned using CLUSTALX 2.0.7 [26], whereas the $r e c A, \sec A$, and $\sec Y$ gene sequences were aligned by positioning their codons on the corresponding protein alignments. To do so, the amino acid sequences from the corresponding gene sequences were first deduced using the bacterial translation table from GETORF in EMBOSS 6.0.1 [27]. They were then aligned using CLUSTALX 2.0.7, and the codons were positioned according to the amino acid alignments. Ambiguous regions in the alignments were filtered out with GBLOCKS 0.91b [28]. A fifth dataset was produced by concatenating the resulting filtered sequences. Bootstrap replicates for the $\mathrm{ML}$ analyses were generated with SEQBOOT from the PHYLIP 3.67 package [29].

\section{Phylogenetic analyses}

Computations and analyses were performed on a cluster of four AMD64 Opteron 875 Dual Core 2.2 Ghz/2 MB L2 cache CPUs with 16 GB of RAM running on Debian 4.0 (kernel 2.6.16.21), a MacBook Pro laptop with a Core 2 Duo 2.4 Ghz/4 MB L2 cache CPU and 2 GB of RAM running on MacOS X 10.5.4, or on an ASUS M6NBe laptop with a $1.6 \mathrm{G} \mathrm{Hz} / 2 \mathrm{MB}$ L2 cache Dothan CPU and $1 \mathrm{~GB}$ of RAM running on MS Windows XP SP3. Maximum likelihood (ML) analyses were computed using PHYML 3.0 [30] under the GTR $+\Gamma 4+\mathrm{I}$ nucleotide substitution model. This model was selected using the Akaike Information Criterion (Akaike 1973), as implemented using jModelTest 3.7 [31]. One hundred bootstrap replicates were performed for each ML analysis. Maximum parsimony (MP) analyses were performed with PAUP* $4.0 \mathrm{~b} 10$ [32], each using a thousand bootstrap replicates.

\section{Accession numbers}

Nucleotide sequence data reported are available in the GenBank database under accession numbers [GenBank: FJ154797] to [GenBank: FJ154838] (Table 2).

\section{Authors' contributions}

JFP designed the study, verified the phenotypic validation of the S. vestibularis strains, sequenced the 16S RNAencoding, $\sec A$, and $\sec Y$ genes with the help of VS, prepared the accession numbers, performed the data mining, sequence alignments and phylogenetic analyses, generated the figures and tables, and drafted the manuscript. VS participated in the 16S RNA-encoding, $\sec A$, and $\sec Y$ gene sequencing and determined the recA gene sequences. $\mathrm{MB}$ coordinated the work of VS and the isolation of CCRI streptococcal strains. MF participated in the design and coordination of the study and helped draft the manuscript. All the authors have read and approved the final manuscript.

\section{Acknowledgements}

This study was funded by Canadian Institute of Health Research (CIHR) operating grant MOP 177248 to MF. JFP is the recipient of the FQRNT/ Génome Québec Louis-Berlinguet postdoctoral fellowship. The sequencing service of the Centre de bio-informatique et de biologie computationnelle (CBBC) at Université Laval and the CHUL sequencing service sequenced the DNA templates. We thank Gene Bourgeau for editorial help and Dr. John R. Tagg for kindly sending us $S$. salivarius strain $\mathrm{KI} 2$.

\section{References}

I. Euzéby JP: List of Bacterial Names with Standing in Nomenclature: a folder available on the Internet. Int J Syst Bacteriol 1997, 47:590-592.

2. Kawamura Y, Hou XG, Sultana F, Miura H, Ezaki T: Determination of I 6S rRNA sequences of Streptococcus mitis and Streptococcus gordonii and phylogenetic relationships among members of the genus Streptococcus. Int J Syst Bacteriol 1995, 45:406-408.

3. Marsh PD: Oral ecology and its impact on oral microbial diversity. In Oral ecology: the molecular basis Edited by: Kuramitsu H, Ellen RP. Wymonham, UK: Horizon Scientific Press; 2000: I I-65.

4. Whiley RA, Hardie JM: Streptococcus vestibularis sp. nov. from the human oral cavity. Int J Syst Bacteriol 1988, 38:335-339.

5. Schleifer KH, Ehrmann M, Krusch U, Neve H: Revival of the species Streptococcus thermophilus (ex Orla-Jensen, 1919) nom. rev. System Appl Microbiol 1991, I4:386-388.

6. Girard F, Lautier M, Novel G: DNA-DNA homology between plasmids from Streptococcus thermophilus. Lait 1987, 67:537-544.

7. Jayarao BM, Pillai SR, Wolfgang DR, Griswold DR, Hutchinson LJ: Herd level information and bulk tank milk analysis: tools for improving milk quality and udder health. Bovine Practitioner 200I, 35:23-37.

8. Bruttin A, Desiere F, d'Amico N, Guerin JP, Sidoti J, et al.: Molecular ecology of Streptococcus thermophilus bacteriophage infections in a cheese factory. Appl Environ Microbiol 1997, 63:3144-3150.

9. Hardie JM, Whiley RA: The Genus Streptococcus--Oral. In The Prokaryotes Third edition. Edited by: Dworkin M, Falkow S, Rosenberg E, Schleifer K-H, Stackebrandt E. Springer; 2006:76-107.

10. Doyuk E, Ormerod OJ, Bowler IC: Native valve endocarditis due to Streptococcus vestibularis and Streptococcus oralis. J Infect 2002, 45:39-4I.

II. Partridge SM: Prosthetic valve endocarditis due to Streptococcus vestibularis. J Infect 2000, $41: 284-285$.

12. Corredoira JC, Alonso MP, Garcia JF, Casariego E, Coira A, et al:: Clinical characteristics and significance of Streptococcus salivarius bacteremia and Streptococcus bovis bacteremia: a 
prospective 16-year study. Eur J Clin Microbiol Infect Dis 2005, 24:250-255.

13. Hols P, Hancy F, Fontaine L, Grossiord B, Prozzi D, et al.: New insights in the molecular biology and physiology of Streptococcus thermophilus revealed by comparative genomics. FEMS Microbiol Rev 2005, 29:435-463.

14. Poyart C, Quesne G, Coulon S, Berche P, Trieu-Cuot P: Identification of streptococci to species level by sequencing the gene encoding the manganese-dependent superoxide dismutase. J Clin Microbiol 1998, 36:4 I-47.

15. Papanikou E, Karamanou S, Economou A: Bacterial protein secretion through the translocase nanomachine. Nat Rev Microbiol 2007, 5:839-85।.

16. Cox MM: Motoring along with the bacterial RecA protein. Nat Rev Mol Cell Biol 2007, 8: I27-I 38.

17. Sapp J: Two faces of the prokaryote concept. Int Microbiol 2006, 9:163-172.

18. Selmer M, Dunham CM, Murphy FVt, Weixlbaumer A, Petry S, et al.: Structure of the $70 \mathrm{~S}$ ribosome complexed with mRNA and tRNA. Science 2006, 313:1935-1942.

19. Janda JM, Abbott SL: I6S rRNA gene sequencing for bacterial identification in the diagnostic laboratory: pluses, perils, and pitfalls. J Clin Microbiol 2007, 45:276I-2764.

20. Gold VA, Duong F, Collinson I: Structure and function of the bacterial Sec translocon. Mol Membr Biol 2007, 24:387-394.

21. Li X, Heyer WD: Homologous recombination in DNA repair and DNA damage tolerance. Cell Res 2008, I8:99-II3.

22. Rasmussen TB, Danielsen M, Valina O, Garrigues C, Johansen E, et al.: Streptococcus thermophilus core genome: comparative genome hybridization study of $\mathbf{4 7}$ strains. Appl Environ Microbiol 2008, 74:4703-47I0.

23. Lévesque CM, Lamothe J, Frenette M: Coaggregation of Streptococcus salivarius with periodontopathogens: evidence for involvement of fimbriae in the interaction with Prevotella intermedia. Oral Microbiol Immunol 2003, 18:333-337.

24. Sambrook J, Fritsch EF, Maniatis T: Molecular cloning: a laboratory manual. Cold Spring Harbor, NY, USA: Cold Spring Harbor Laboratory Press; 1989.

25. Pombert JF, Otis C, Lemieux C, Turmel M: The complete mitochondrial DNA sequence of the green alga Pseudendoclonium akinetum (Ulvophyceae) highlights distinctive evolutionary trends in the chlorophyta and suggests a sister-group relationship between the Ulvophyceae and Chlorophyceae. Mol Biol Evol 2004, 21 :922-935.

26. Larkin MA, Blackshields G, Brown NP, Chenna R, McGettigan PA, et al.: Clustal $\mathbf{W}$ and Clustal $\mathbf{X}$ version 2.0. Bioinformatics 2007, 23:2947-2948.

27. Rice P, Longden I, Bleasby A: EMBOSS: the European Molecular Biology Open Software Suite. Trends Genet 2000, 16:276-277.

28. Castresana J: Selection of conserved blocks from multiple alignments for their use in phylogenetic analysis. Mol Biol Evol 2000, I 7:540-552.

29. Felsenstein J: PHYLIP - Phylogeny Inference Package (Version 3.2). Cladistics 1989, 5:164-166.

30. Guindon S, Gascuel O: A simple, fast, and accurate algorithm to estimate large phylogenies by maximum likelihood. Syst Biol 2003, 52:696-704.

31. Posada D: jModelTest: Phylogenetic model averaging. Mol Biol Evol 2008, 25: I253-I256.

32. Swofford DL: PAUP* Phylogenetic Analysis Using Parsimony (* and other methods). In Version 4.0b/0 ed Sunderland, MA, USA: Sinauer Associates; 2003.
Publish with Biomed Central and every scientist can read your work free of charge

"BioMed Central will be the most significant development for disseminating the results of biomedical research in our lifetime. "

Sir Paul Nurse, Cancer Research UK

Your research papers will be:

- available free of charge to the entire biomedical community

- peer reviewed and published immediately upon acceptance

- cited in PubMed and archived on PubMed Central

- yours - you keep the copyright
BioMedcentral 